\title{
MicroRNA-643 promotes proliferation and inhibits apoptosis of papillary thyroid carcinoma by down-regulating the cytochrome P450 family member 11B1
}

\author{
Hongqing Yin, Jun Shao \\ Department of Ultrasonography, Affiliated Kunshan Hospital of Jiangsu University, Suzhou 215300, China \\ Contributions: (I) Conception and design: J Shao; (II) Administrative support: None; (III) Provision of study materials or patients: H Yin; (IV) \\ Collection and assembly of data: H Yin; (V) Data analysis and interpretation: All authors; (VI) Manuscript writing: All authors; (VII) Final approval \\ of manuscript: All authors. \\ Correspondence to: Jun Shao. Department of Ultrasonography, Affiliated Kunshan Hospital of Jiangsu University, No. 91, Qianjin West Road, \\ Kunshan, Suzhou 215300, China. Email: shaojun18913201188@163.com.
}

Background: Thyroid cancer is the most common malignant tumor in endocrine system. Papillary thyroid carcinoma (PTC), accounting for 60-70\% of all thyroid cancer cases, is the most common type of thyroid cancer. Nowadays, the treatments for PTC are limited and the prognosis is poor. Exploring the underlying mechanism of PTC development and finding evidence for molecular targeted therapy have always been urgent problems. The purpose of the current study is to investigate the clinical value of microRNA (miR)-643, and its related mechanism regulating PTC proliferation and apoptosis by regulating cytochrome P450 family member 11b1 (CYP11B1).

Methods: Forty-two confirmed human PTC tissue specimens, corresponding adjacent normal thyroid tissue specimens, and serum samples were collected from September 2018 to April 2019. The transfected cell lines were divided into four groups: control group, empty group, si-miR-643 group, and si-miR-643 + si-CYP11B1 group. Real-time quantitative PCR (qRT-PCR) was used to detect the relative expression of miR-643 and CYP11B1 mRNA. The expression of CYP11B1 protein was detected by Western blot (WB). Cell proliferation was detected by cell counting kit-8 (CCK-8) and colony formation assay. Cell apoptosis was detected by flow cytometry. The dual-luciferase reporter gene assay was used to verify the targeting relationship between miR-643 and CYP11B1.

Results: Compared to adjacent non-tumor tissues, miR-643 expression in PTC tissues was significantly up-regulated $(\mathrm{P}=0.019)$. Pearson correlation analysis showed that miR-643 level in serum was significantly correlated with that in PTC tissue $\left(r_{\text {Pearson }}=0.546, \mathrm{P}<0.001\right)$. MiR-643 expressions in both PTC tissue and serum were significantly associated with tumor size and histological grading $(\mathrm{P}<0.05)$. Patients with larger diameter tumors or moderate-poorly differentiated tumors were more likely to have higher miR-643 expression levels in both PTC tissue and serum. CYP11B1 was indicated to be an important downstream molecule of miR-643 by the results of online bioinformatics prediction software Targetscan and the luciferase reporter gene assay. Compared with the control group and empty group, the apoptotic ability of cells in si-miR-643 group increased significantly $(\mathrm{P}<0.05)$, while cell proliferation in si-miR-643 group was inhibited significantly $(\mathrm{P}<0.05)$. Further research showed that small interference RNA (siRNA)-mediated miR-643 silencing induced a notable reduction of antigen KI67 (ki-67) expression and a dramatic elevation of CYP11B1, BCL2-associated X protein (Bax) and caspase-3 expressions in TPC-1 cells in comparison with that in the control group and empty group, while CYP11B1 knockdown markedly reversed the above phenomenon.

Conclusions: MiR-643 can promote the proliferation of TPC-1 cells and inhibit its apoptosis through regulating CYP11B1. 


\begin{abstract}
Keywords: Papillary thyroid carcinoma (PTC); microRNA (miRNA); cytochrome P450 family member 11B1 (CYP11B1); proliferation; apoptosis
\end{abstract}

Submitted Sep 25, 2019. Accepted for publication Jan 06, 2020.

doi: $10.21037 /$ tcr.2020.01.43

View this article at: http://dx.doi.org/10.21037/tcr.2020.01.43

\section{Introduction}

Thyroid cancer is the most common malignant tumor in endocrine system, accounting for one-third of all head and neck cancers $(1,2)$. In recent years, the incidence of thyroid cancer has continued to rise. According to the 2018 Global Cancer Statistics Report, 567,233 new cases of thyroid cancer are expected to be diagnosed in 2018, and 41,071 cases of thyroid cancer death (3). Thyroid cancer mainly originates from cells of different origins in the thyroid gland and is divided into four pathological types, namely papillary carcinoma, follicular carcinoma, undifferentiated carcinoma and medullary carcinoma (4). Great variability has been observed among different pathological types (4). The most common type is papillary thyroid carcinoma (PTC), which accounts for $60-70 \%$ of all thyroid cancer cases (5). The onset and development of PTC are generally hidden. Most patients have no obvious symptoms or signs in early stage, and it can only be detected by physical examination (6). Patients have obvious symptoms in advanced stage due to excessive tumor growth, local invasion or distant metastasis (6). However, the use of traditional treatments is limited for patients in advanced stages, which contributes to the poor prognosis of PTC. Therefore, exploring the underlying mechanism of PTC development and finding evidence for molecular targeted therapy have always been urgent problems.

Although the pathogenesis of thyroid cancer is not fully understood, it is generally considered to be the interaction between innate genes and acquired environment $(7,8)$. Researchers have discovered many genetic factors involved in the onset of this disease. As a single-chain, highly conserved, non-coding small RNA, microRNA (miRNA) is closely related to human diseases including tumors (9). MiRNAs were originally thought to be a negative regulator and play an important role in regulating gene expression. MiRNAs mainly bind to regions within the 3'UTR of the target messenger RNA in a sequence-complementary manner to exert their effects (9). However, recent research data suggested that miRNA regulation has a more complex post-transcriptional control system than the originally recognized theory (9). Studies have confirmed that the development of tumors was related to the dysregulation of miRNAs (10). The abnormal expressions of miRNAs have been proven to be involved in the process of tumor differentiation, proliferation, and apoptosis. Recent studies have reported that miRNAs could serve as supplementary therapeutic targets for thyroid tumors (11), suggesting that the functions of these small non-coding RNAs are worthy to be explored.

MiR-643 is a member of a family of miRNA precursors firstly found in mammals (12). A study has shown that miR643 was associated with pancreatic cancer development (13). However, the role and mechanism of miR-643 in PTC are not clear yet. This study was carried out in the following aspects: (I) comparing miR-643 expressions in PTC tissues and matched paracancerous tissues; (II) predicting and verifying the target gene of miR-643; (III) exploring the biological function of miR-643 in PTC development. The purpose of this study is to explore a new theoretical target for PTC treatment.

\section{Methods}

\section{Source of materials used in this study}

Human PTC cell lines TPC-1 (Lot number: CS137-0022), K1 (Lot number: CS137-0015), BCPAP (Lot number: CS137-0011), GLAG-66 (Lot number: CS137-0016), KTC-3 (Lot number: CS137-0026) and human thyroid normal cells Nthy-ori 3-1 (Lot number: CS137-0005) were purchased from the Cell Resource Center of Shanghai Institutes for Biological Sciences, Chinese Academy of Sciences. Dulbecco's Modified Eagle's medium (DMEM, Lot number: Z1800-22) and real-time quantitative PCR (qRT-PCR) MIX (Lot number: U1805-39) were purchased from Shanghai Jietai Biotechnology Company. Total RNA Isolation (TRIZOL) kit (Lot number: 201807038), Lipofectamine 2000 reagent (Lot number: L18-34110001) and annexin V fluorescein isothiocyanate (FITC)/ 
propidium iodide (PI) apoptosis assay kit (Lot number: X18-0144-0300) were purchased from Invitrogen, USA. Cell culture flasks, dimethyl sulfoxide (DMSO, Lot number: 2018012307) and radio-immunoprecipitation assay (RIPA) lysates (Lot number: 2018021417) were purchased from Wuhan Huamei Bioengineering Co., Ltd. Fetal bovine serum (Lot number: B18-1185) was purchased from Beijing Aoke Biotechnology Co., Ltd. Primer synthesis was provided by Beijing Qingke New Industry Biotechnology Co., Ltd. antigen KI67 (ki-67, rabbit monoclonal, 1:2,000; Lot number: Ab18-1506-021), BCL2-associated X protein (Bax, rabbit monoclonal, 1:3,000; Lot number: Ab18-8732093), caspase-3 (rabbit monoclonal, 1:2,000; Lot number: Ab18-6629-008), CYP11B1 (rabbit monoclonal, 1:1,000; Lot number: Ab18-7836-106) and glyceraldehyde-3phosphate dehydrogenase (GAPDH, rabbit monoclonal, 1:4,000; Lot number: Ab18-0132-342) primary antibodies and secondary antibodies (anti-rabbit, 1:3,000; Lot number: Ab18-4396-881) were purchased from Abcam, UK. The RNA extraction kit (Lot number: 0181004) was purchased from Bioteke, China.

\section{Collection of PTC tissues, adjacent non-tumor tissues, and fast blood samples}

PTC was diagnosed by intraoperative pathological biopsy. Forty-two paired human PTC tissue specimens and adjacent normal thyroid tissue specimens (at least $3 \mathrm{~cm}$ far from the edge of the tumor tissue) were collected from September 2018 to April 2019. Fast blood samples were collected in the morning of surgery. All patients were diagnosed as PTC for the first time with no other tumor history and received no anti-tumor treatment before. The clinical specimens were provided by the Affiliated Kunshan Hospital of Jiangsu University. Informed consent was obtained from every patient in accordance with the Declaration of Helsinki. The study was approved by the Hospital Medical Ethics Committee. The ethical approval number is EA20180105.

\section{Cell culture and cell transfection}

TPC-1, K1, BCPAP, GLAG-66, KTC-3 and Nthy-ori 3-1 cell lines were cultured in DMEM medium supplemented with $10 \%$ fetal bovine serum. The culture conditions were $37{ }^{\circ} \mathrm{C}$ with $5 \% \mathrm{CO}_{2}$. Small interference RNAs (siRNAs) targeting miR-643 (si-miR-643) and si-CYP11B1 were obtained from GenePharma Co. Ltd. (Suzhou, China, Lot number: W-19-09602). Cells were divided into four groups: control group, empty group, si-miR-643 group, and si-miR-643 + si-CYP11B1 group. The transfection was performed using Lipofectamine 2000 according to the instruction.

\section{$R N A$ extraction, reverse transcription and $q R T-P C R$}

Serum total RNA was extracted using the RNA extraction kit, then was transcribed into cDNA. The expressions of miR-643 and CYP11B1 were determined using qRTPCR. The reaction system was as follows: $10 \mu \mathrm{L}$ of SYBR ${ }^{\circledR}$ Premix, $1 \mu \mathrm{L}$ of each of the upstream and downstream primers, $2 \mu \mathrm{L}$ of the DNA template, and $6 \mu \mathrm{L}$ of $\mathrm{ddH}_{2} \mathrm{O}$. After denaturation at $95{ }^{\circ} \mathrm{C}$ for $5 \mathrm{~min}, 42$ cycles were carried out (circulation conditions: $95{ }^{\circ} \mathrm{C}$ for $30 \mathrm{~s}, 57^{\circ} \mathrm{C}$ for $30 \mathrm{~s}$, and $72{ }^{\circ} \mathrm{C}$ for $30 \mathrm{~s}$ ). U6 and GAPDH were used as endogenous controls for miR-643 and CYP11B1, respectively. Primer sequences are as follows: $\mathrm{miR}$ 643 forward: 5'-TGCAGTCAGTGCAGGTAG-3', reverse: 5'-GAACAGTTCTGCGTATCTC-3'; U6 forward: 5'-CTCGCTTCGGCAGCACA-3', reverse: 5 '-AACGCTTCACGAATTTGCGT-3 '; CYP11B 1 forward: 5'-TACCGATCGAAGTCG-3', reverse: 5 '-GTTCCCACTAGGGCTAAGTC-3 '; GAPDH forward: 5 ' -CTCGCTTCGGCAGCACAG-3 ', reverse: 5'-ATCGATTTGATCGAGGGT-3'. Relative quantification was performed using the $2^{-\Delta \Delta \mathrm{Ct}}$ method. All tests were performed in triplicate.

\section{Western blot (WB) assay}

Cellular proteins were extracted using RIPA lysis buffer and protein concentration was measured using the BCA kit. After isolating the protein on a $10 \%$ SDS gel, proteins were transferred to PVDF membranes and were incubated with CYP11B1, ki-67, Bax, caspase-3, or GAPDH primary antibodies overnight at $4{ }^{\circ} \mathrm{C}$. Then the membranes were incubated with the secondary antibody for another $2 \mathrm{~h}$ at room temperature. Protein expression levels were finally qualified using enhanced chemiluminescence (ECL) reagents.

\section{Cell counting kit-8 (CCK-8) assay for cell proliferation}

When the PTC cell confluence reached about $80 \%$, the cells were washed twice in PBS and digested with $0.25 \%$ trypsin to form a single cell suspension. The cells in the suspension were counted, and $3 \times 10^{3}-6 \times 10^{3}$ cells were seeded 
in 96-well plates $(200 \mu \mathrm{L} /$ well $)$, and the corresponding plasmids were transfected and placed at $37^{\circ} \mathrm{C}$ with $5 \% \mathrm{CO}_{2}$. The culture is carried out in a saturated humidity incubator. The plates were taken at different time periods $(0,24,48$ and $72 \mathrm{~h}$ ), and $10 \mu \mathrm{L}$ of CCK-8 solution was added to each well and cultured for $2 \mathrm{~h}$. The optical density (OD) value at a wavelength of $570 \mathrm{~nm}$ was measured using an enzymelinked immunosorbent assay. All tests were performed in triplicate.

\section{Colony formation assay}

Stably transfected PTC cells were seeded in 6-well plates (500 cells/well) and cultured in an incubator with $5 \% \mathrm{CO}_{2}$ at $37^{\circ} \mathrm{C}$ for about 2 weeks. When clear colonies formed, the cells were washed with PBS and fixed by $70 \%$ ethanol for 3 min. Then, the plates were stained with $1 \%$ crystal violet for $30 \mathrm{~min}$. The number of colonies was counted.

\section{Flow cytometry for apoptosis}

Each group of cells was digested with trypsin to prepare a single cell suspension. After mixed with $5 \mu \mathrm{L}$ of annexin $\mathrm{V}$-FITC fluorescent dye, it was incubated for $15 \mathrm{~min}$ at room temperature. Then, $5 \mu \mathrm{L}$ of PI fluorescent dye was added, and the apoptosis of each group was observed at $488 \mathrm{~nm}$.

\section{Dual-luciferase reporter gene assay}

MiR-643 mimics (5'-UUAAUCACUUGAUACUGA-3') and CYP11B1 3'UTR (upstream: 5'-GCCATCGAGC CTCACGGCTA-3'; downstream: 5'-GAGCTCCGAG ATCCTAGACAT-3') were transferred to the pmiR-RBReport $^{\mathrm{TM}}$ reporter gene (Beijing Huaketai Biotechnology Co., Ltd.). Luciferase reporter plasmids (CYP11B1 wild type and mutant) were co-transfected into PTC cells with miR-643, respectively. The luciferase activity was detected by a luciferase reporter assay kit. All tests were performed in triplicate.

\section{Statistical analysis}

Statistical analysis and charting were performed using SPSS 20.0 and GraphPad Prism 5.0 software. The comparison between the count data groups was performed using $\chi^{2}$ test. Measurement data are expressed as mean \pm standard deviation. Correlation analysis was performed using the Pearson method. Comparison between groups was performed by $\mathrm{F}$ test or $t$-test. $\mathrm{P}<0.05$ was considered as statistically significant.

\section{Results}

MiR-643 expression in PTC tissue was significantly bigher than that in adjacent non-tumor tissue and was positively correlated with serum miR-643 levels

Forty-two patients consisting of 13 male and 29 female were included in our study. PTC tissues, adjacent non-tumor tissues, and corresponding serum samples were collected, and expression levels of miR-643 were detected by qRT-PCR. The melting peaks of miR-643 (Figure $1 A$ ) and U6 (Figure $1 B$ ) were single, indicating that the primers did not form primer dimers and there was no non-specific amplification. For PTC tissue, adjacent nontumor tissue, and corresponding serum samples, miR643 expression levels were $5.95 \pm 0.78,3.37 \pm 0.59$ and $4.47 \pm 1.03$, respectively. Compared with adjacent nontumor tissue, miR-643 expression level in PTC tissue was significantly up-regulated $(\mathrm{P}=0.019$, Figure $1 C)$. Pearson correlation analysis showed that miR-643 level in serum was significantly correlated with that in PTC tissue $\left(r_{\text {Pearson }}\right.$ $=0.546, \mathrm{P}<0.001$, Figure $1 D)$, but had no association with that in adjacent non-tumor tissue $\left(r_{\text {Pearson }}=-0.075, \mathrm{P}=0.635\right.$, Figure 1E).

\section{MiR-643 expression in both PTC tissue and serum were significantly associated with tumor size and bistological grading}

We further explored the relationships between miR643 expression levels and patients' clinical pathological characteristics (Table 1). We found that miR-643 expression levels had no significant associations with gender, age, smoking, and drinking (all $\mathrm{P}>0.05$ ). However, miR-643 expression levels in both PTC tissue and serum were significantly associated with tumor size and histological grading $(\mathrm{P}<0.05)$. Patients with larger diameter tumors or with moderate-poor differentiated were likely to have higher miR-643 expression levels in both PTC tissue and serum.

\section{Cell line screening and validation of miR-643 transfection}

As shown in Figure 2A, miR-643 expression was significantly increased in TPC-1, K1, BCPAP, GLAG-66, and KTC-3 cell lines compared to that in Nthy-ori 3-1 cell 

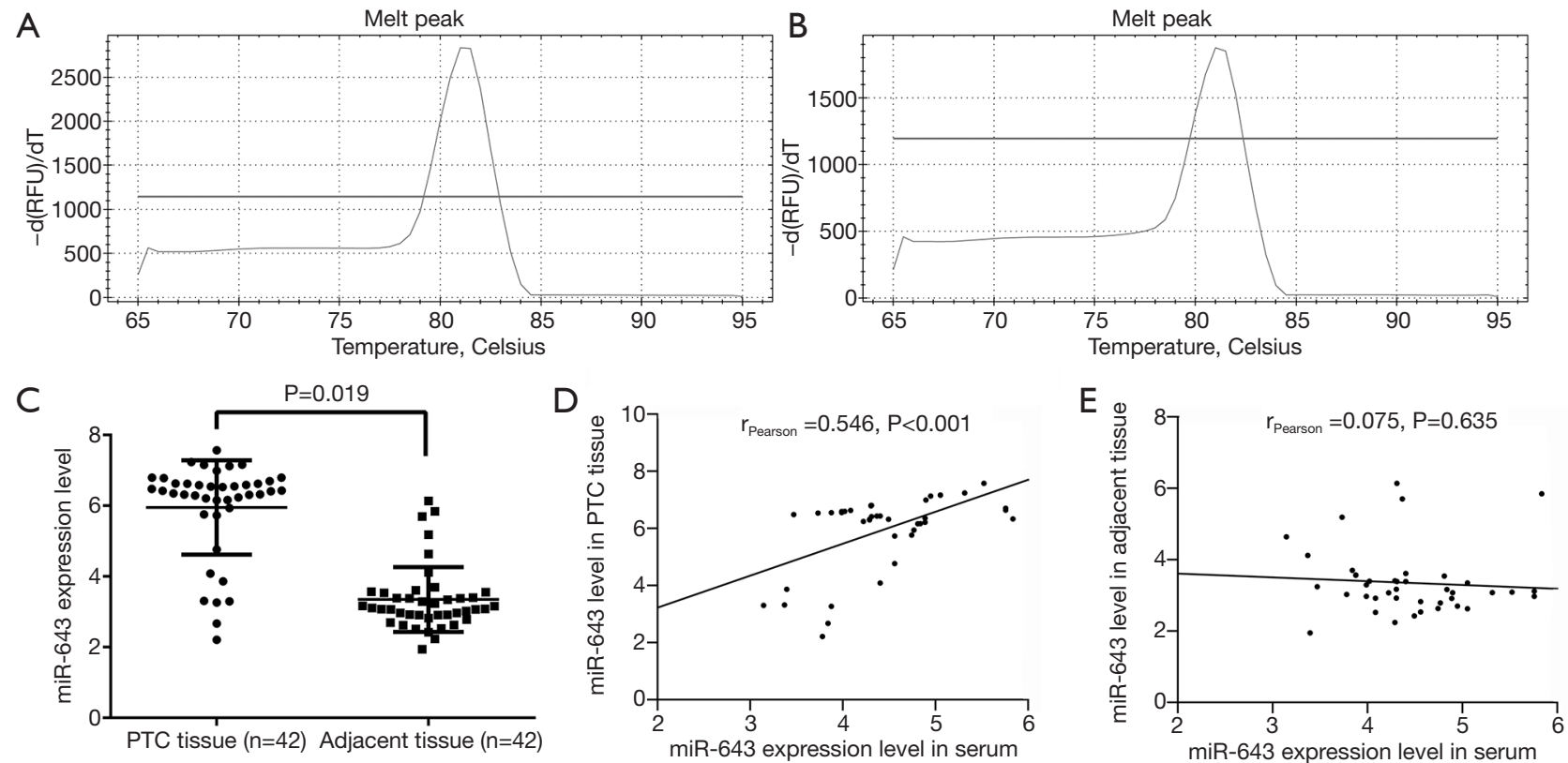

Figure 1 MiR-643 expression in papillary thyroid carcinoma (PTC) tissue, non-tumor tissue, and serum. (A) The melting peak of miR643; (B) the melting peak of U6; (C) miR-643 expression level in PTC tissue was significantly higher than that in adjacent non-tumor tissue; (D) miR-643 level in PTC tissue was significantly correlated with that in serum; (E) miR-643 level in adjacent non-tumor tissue was not correlated with serum.

Table 1 Relationships between miR-643 expression and patients' clinical pathological characteristics (data are presented as mean \pm standard deviation)

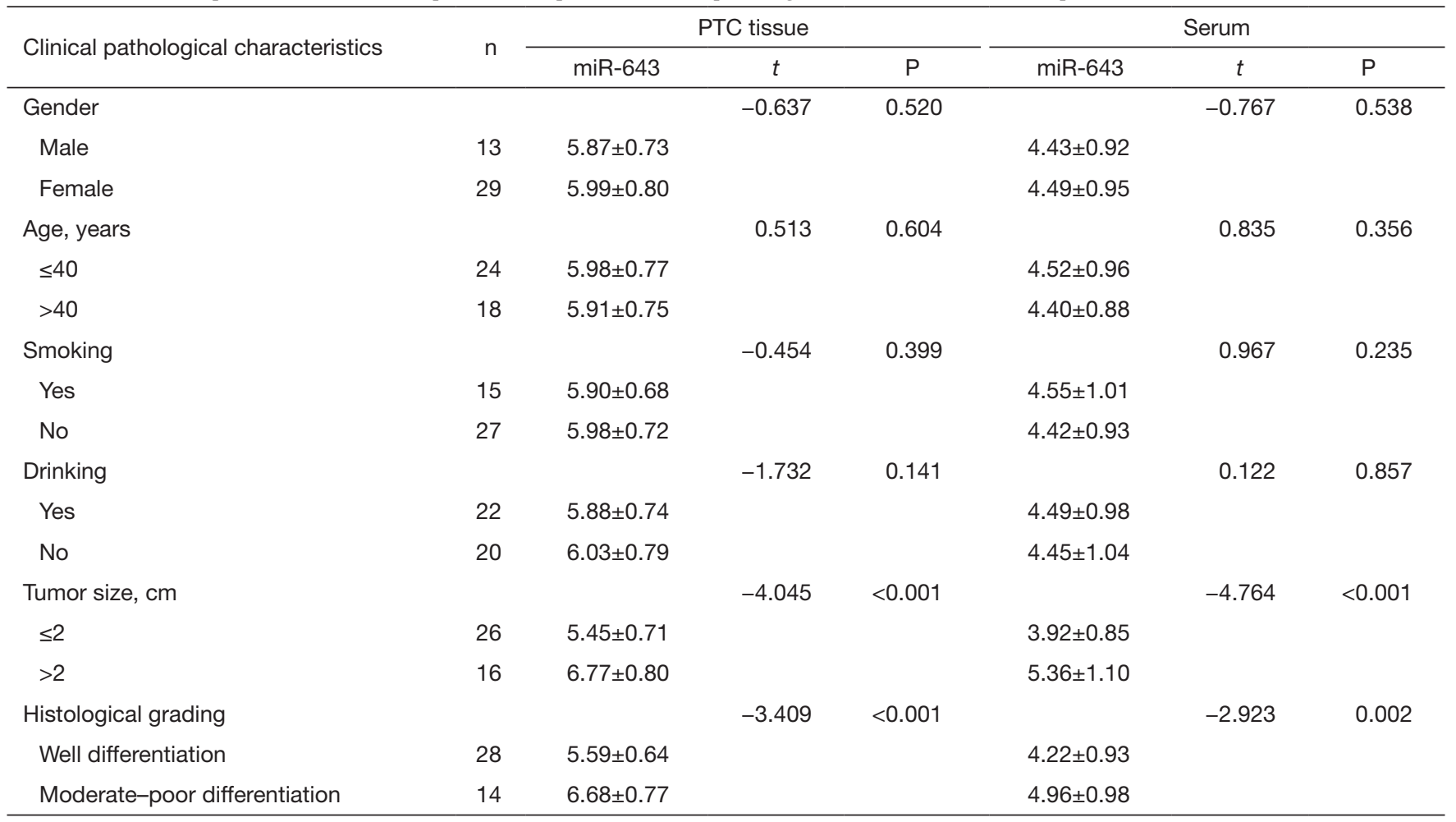

PTC, papillary thyroid carcinoma. 

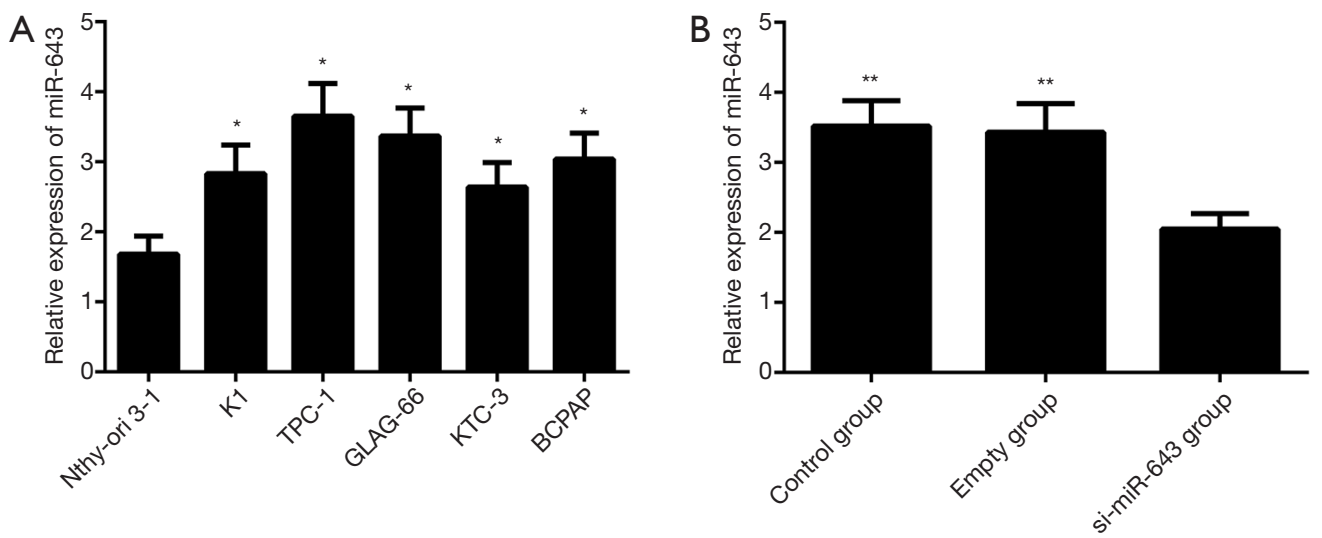

Figure 2 Cell line screening and validation of miR-643 transfection. (A) MiR-643 expression was significantly increased in the TPC-1, K1, BCPAP, GLAG-66 and KTC-3 cell lines compared to the Nthy-ori 3-1 cell line; (B) compared with the control group and empty group, miR-643 expression was significantly inhibited after transfection with miR-643 inhibitor. *, compared with Nthy-ori 3-1 cell line, $\mathrm{P}<0.05$; **, compared with si-miR-643 group, $\mathrm{P}<0.01$.

line. Since miR-643 expressed most highly in TPC-1 cell line, this cell line was selected for subsequent transfection experiments. The validation of si-miR-643 transfection was shown in Figure 2B. There was significant difference in miR-643 expressions among control group, empty group, and si-miR-643 group $(\mathrm{P}=0.003)$. Compared with the control group and empty group, miR-643 expression was significantly decreased after transfection (all $\mathrm{P}<0.05$ ), but there was no significant difference in miR-643 expressions between the control group and empty group $(\mathrm{P}>0.05)$.

\section{Prediction and verification of the targeting relationship between miR-643 and CYP11B1}

Online bioinformatics prediction software Targetscan was used to predict miR-643 downstream targets, and CYP11B1 was considered to be an important downstream target of miR-643 (Figure 3A). Luciferase reporter gene assay showed that the luciferase activity of the miR-643 positive group was significantly lower than that of the negative control group in the CYP11B1 wild type cells $(\mathrm{P}<0.05)$, but there was no significant difference in the CYP11B1 mutant cells $(\mathrm{P}>0.05$, Figure 3B). The results of WB and qRT-PCR showed that the protein and mRNA levels of CYP11B1 in si-miR-643 group were significantly higher than those in the empty group and the control group, as shown in Figure 3C,D,E. The melting peaks of CYP11B1 and GAPDH were single (Figure $3 F$ ), indicating that the primers did not form primer dimers and there was no non-specific amplification.

\section{MiRNA-643 promoted proliferation and inbibited apoptosis of PTC by down-regulating CYP11B1}

The effect of miR-643 on apoptosis of TPC- 1 cells was shown in Figure 4A,B. The difference in apoptosis rates among the control group, empty group, si-miR-643 group, and si-miR-643 + si-CYP11B1 group was statistically significant $(\mathrm{P}<0.05)$. Compared with the control group and empty group, the apoptosis of the si-miR-643 group was significantly increased $(\mathrm{P}<0.05)$, while there was no significant difference in the apoptosis rates between empty group and the normal growth group $(\mathrm{P}>0.05)$. Moreover, restoration experiments clarified that CYP11B1 knockdown markedly reversed si-miR-643 induced apoptosis. The effect of miR-643 on the proliferation of TPC-1 cells was shown in Figure $4 C$. There was no significant difference in cell proliferation between the control group, empty group and si-miR-643 group at $0 \mathrm{~h}(\mathrm{P}>0.05)$. However, compared with the control group and empty group, the proliferation of cells in si-miR-643 group was significantly inhibited at 24,48 and $72 \mathrm{~h}(\mathrm{P}<0.05)$, while there was no significant difference in cell proliferation between the control group and empty group $(\mathrm{P}>0.05)$. Moreover, restoration experiments clarified that CYP11B1 knockdown markedly reversed si-miR-643 induced anti-proliferation. The above results were further verified by the colony formation experiment (Figure $5 A, B$ ). Results of WB showed that siRNA-mediated miR-643 silence induced a notable reduction of ki-67 expression and a dramatic elevation of CYP11B1, Bax and caspase- 3 expressions in TPC-1 cells in comparison with that in the 


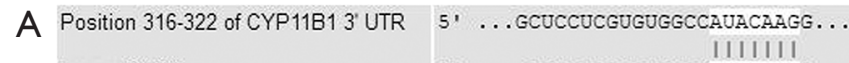
hsa-miR-643 3. GauggacucgaucguauguUCa

B
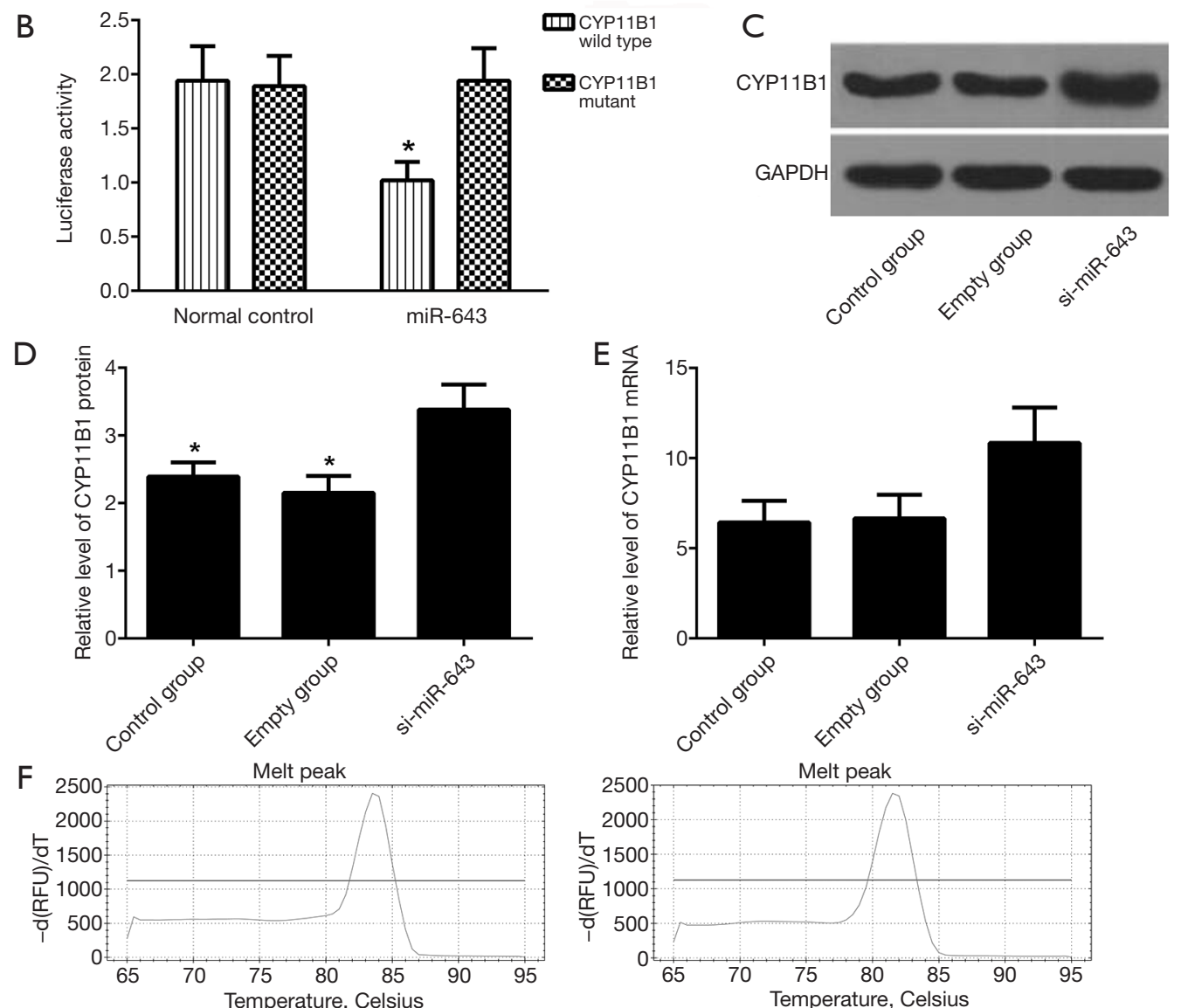

CYP11B1

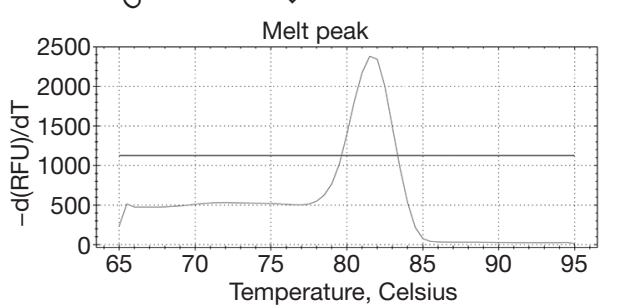

GAPDH

Figure 3 Prediction and verification of the targeting relationship between miR-643 and CYP11B1. (A) CYP11B1 was predicted as a downstream molecule of miR-643; (B) luciferase reporter gene assay showed that the luciferase activity of the miR-643 positive group was significantly lower than that of the negative control group in the CYP11B1 wild type; $(\mathrm{C}, \mathrm{D}, \mathrm{E})$ The protein and mRNA levels of CYP11B1 in the miR-643 inhibitor group were significantly higher than those in the empty group and the normal growth group; (F) the melting peaks of CYP11B1 and GAPDH. *, compared with CYP11B1 mutant group/si-miR-643 group, P<0.05. CYP11B1, cytochrome P450 family member $11 \mathrm{~b} 1$.

control group and empty group. Restoration experiments clarified that CYP11B1 knockdown markedly reversed the above phenomenon (Figure 5C,D,E,F,G). These data indicated that miR-643 affected proliferation and apoptosis of TPC-1 cells partly through regulating CYP11B1.

\section{Discussion}

Thyroid cancer is one of the most common malignant tumors of endocrine system, while PTC is the most common type of thyroid cancer and can occur at any age (1). It is more common in children and young (under 40 years old) women, especially people who had neck X-ray treatment during childhood $(14,15)$. The tumor grows slowly and has no significant symptoms for several years (16) even if the lesion has spread from the primary part of the gland to the other parts of the gland or the cervical lymph nodes (17), so it is easy to ignore its nature. The incidence of PTC is increasing year by year. The conventional treatments of PTC are divided into surgical treatment, ${ }^{131} \mathrm{I}$ internal 

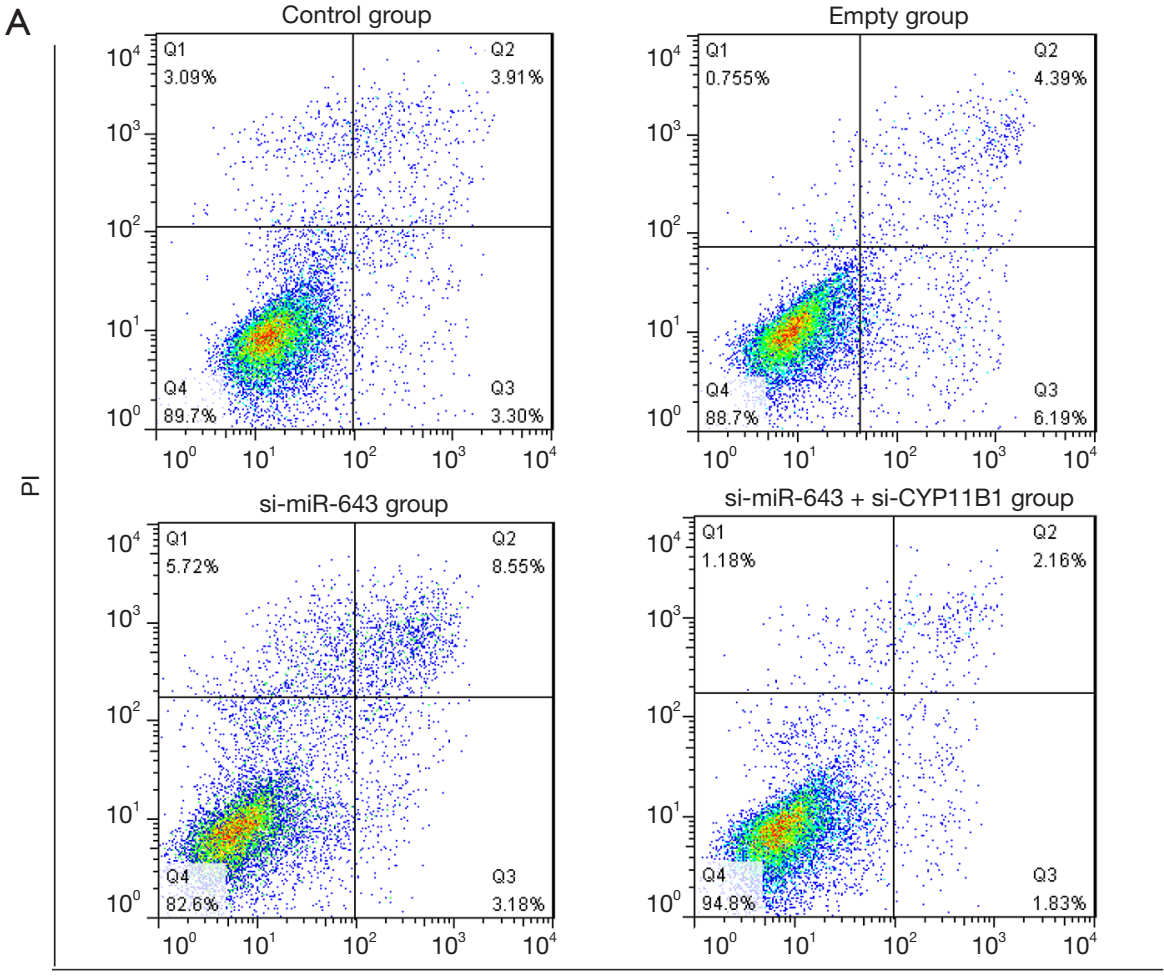

Annexin V-FITC
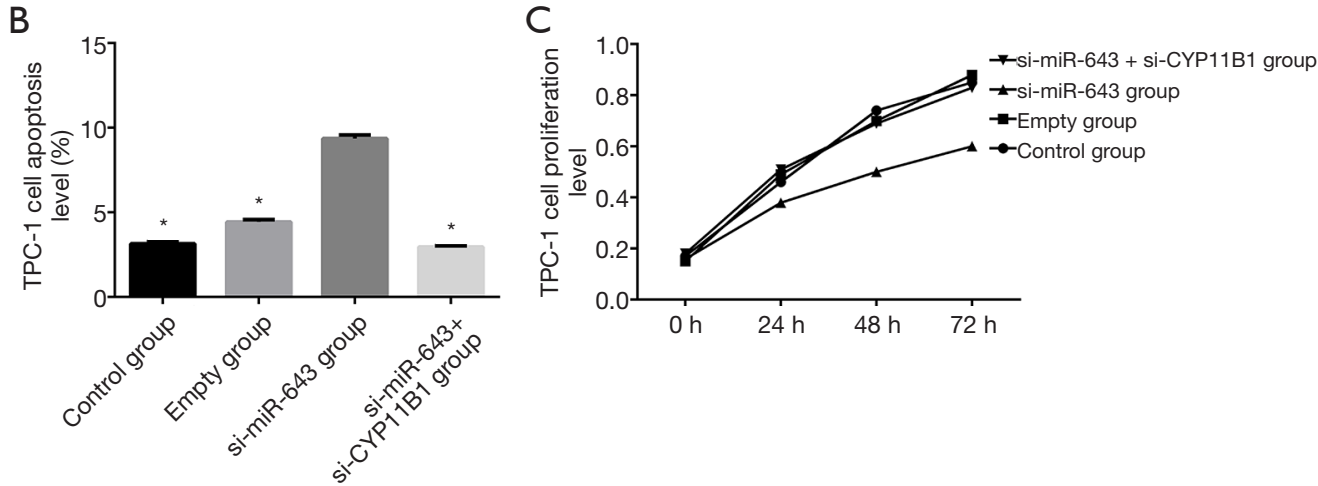

Figure 4 MiRNA-643 promotes proliferation and inhibits apoptosis of PTC by down-regulating CYP11B1: based on flow cytometry and CCK-8 assays. (A,B) Compared with the control group and empty group, the apoptotic ability of the si-miR-643 group was significantly increased $(\mathrm{P}<0.05)$, but there was no significant difference in the apoptosis ability between the empty group and the normal growth group $(\mathrm{P}>0.05)$; (C) at 24, 48 and $72 \mathrm{~h}$, the proliferation of si-miR-643 group was significantly inhibited compared with the control group and empty group $(\mathrm{P}<0.05)$. *, compared with si-miR-643 group, $\mathrm{P}<0.05$. PTC, papillary thyroid carcinoma; CYP11B1, cytochrome $\mathrm{P} 450$ family member 11b1; FITC, fluorescein isothiocyanate.

radiation therapy and endocrine inhibition therapy (18). However, the prognosis is not optimistic due to the lack of early diagnostic methods, and most patients were diagnosed in advanced stage (6). Exploring the underlying mechanism of PTC development, and finding new therapeutic targets have always been urgent problems.
MiRNA plays an important role in the development of various tumors and has become a research hotspot and key point in molecular biology in recent years (19). MiRNA mainly plays the role of mRNA silence, and thereby regulates the expression of target protein, producing a series of molecular biological effects (20). A single miRNA can 


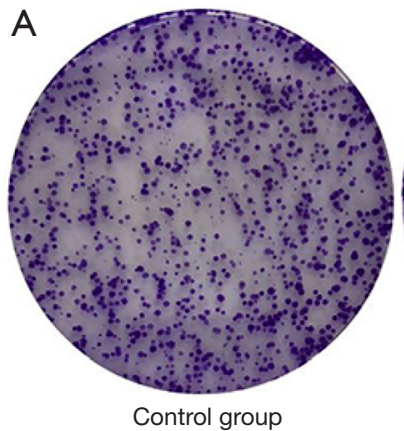

B
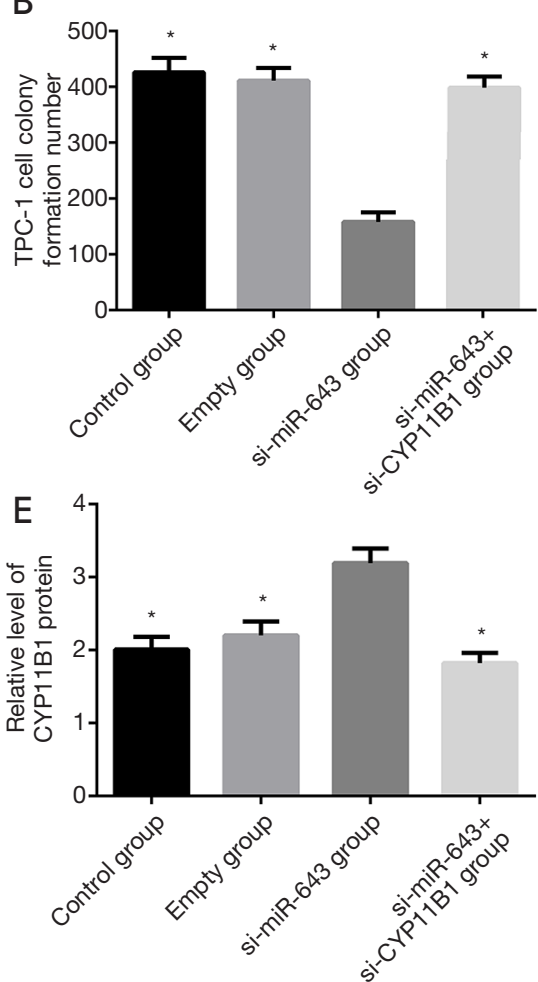

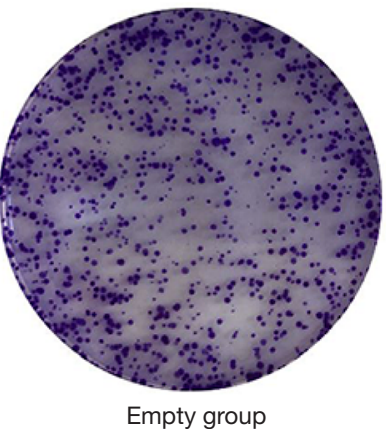

C

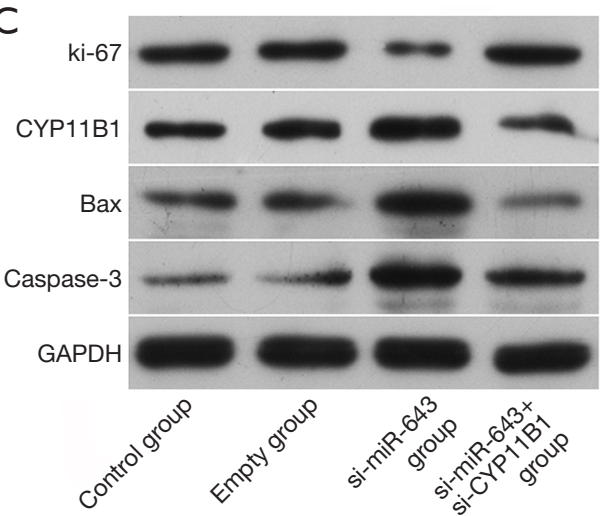

$\mathrm{F}$
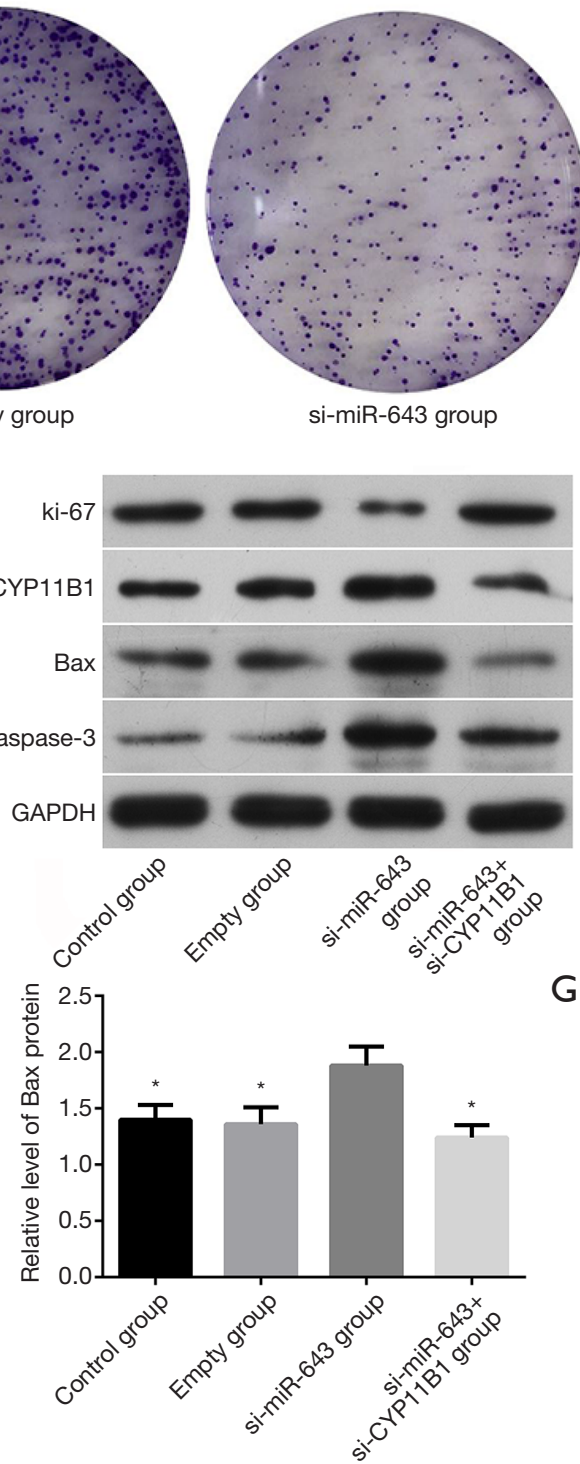

si-miR-643 group

G
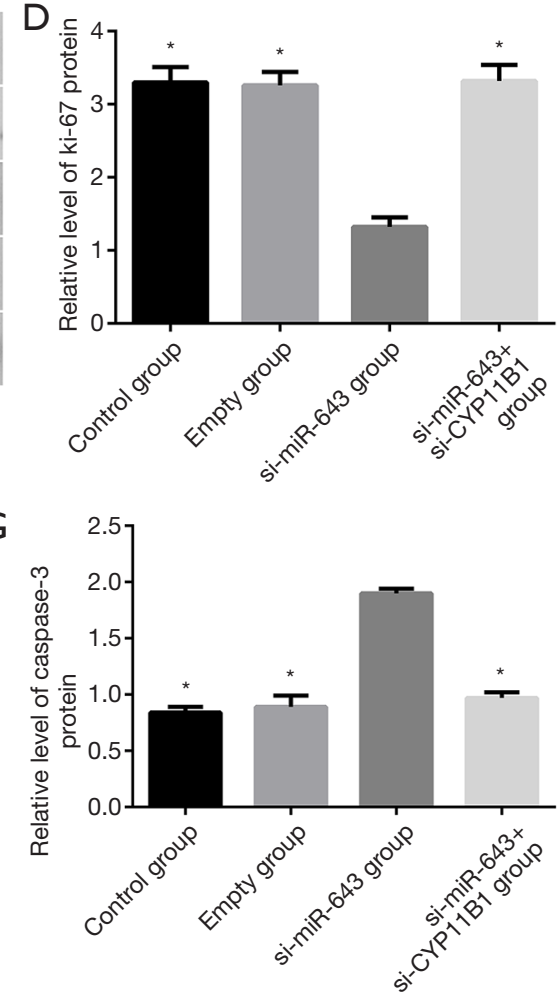

Figure 5 MiRNA-643 promotes proliferation and inhibits apoptosis of PTC by down-regulating CYP11B1: based on colony formation and Western blot assays. (A,B) The proliferation of si-miR-643 group was significantly inhibited compared with the control group and empty group by colony formation experiment (magnification 40x); (C,D,E,F,G) siRNA-mediated miR-643 silencing induced a notable reduction of ki-67 expression and a dramatic elevation of CYP11B1, Bax and caspase-3 in TPC-1 cells in comparison with that in the control group and empty group. However, restoration experiments clarified that CYP11B1 knockdown markedly reversed the above phenomenon. *, compared with si-miR-643 group, $\mathrm{P}<0.05$. CYP11B1, cytochrome P450 family member 11b1; PTC, papillary thyroid carcinoma.

regulate a large number of target mRNAs (20). At present, there is no report on miR-643 and PTC. The current study showed that compared with healthy subjects, the expression of miR-643 in PTC patients' cancer tissues was significantly higher than that in adjacent tissues, and serum miR-643 level was highly correlated with that in cancer tissues, suggesting that we can monitor the PTC well by detecting serum miR-643. In addition, miR-643 levels in serum and cancer tissues were both significantly associated with tumor size and histological grading, suggesting that miR-643 dysregulation is closely associated with PTC tumor progression. At cellular level, we found that downregulation of miR-643 inhibited TPC-1 cell proliferation and promoted apoptosis in TPC-1 cells. We predicted the 
miR-643 target gene by bioinformatics software for the first time. The results of Targetscan and dual-luciferase reporter assays showed that miR-643 could directly target the $3^{\prime}$ non-coding region of CYP11B1 mRNA, thereby inhibiting the expression of CYP11B1. As a tumor suppressor gene, CYP11B1 has been shown to inhibit cancer cell proliferation and promote apoptosis in various solid tumors such as kidney cancer, lung cancer, colorectal cancer, prostate cancer and liver cancer $(21,22)$. In this study, we found that CYP11B1 is a direct target of miR-643, suggesting that in-depth study of the role and mechanism of CYP11B1 in PTC development may provide a new target for PTC therapy.

It is worth noting that this study still has the following shortcomings: (I) the number of PTC patients included in this study is still limited and the patients are only one hospital, which may cause certain bias to the results. Therefore, further multi-center and large-sample prospective cohort studies are highlighted; (II) this study is pending development of PTC animal models to explore a more in-depth exploration of the value of miR-643 in vivo.

\section{Conclusions}

Our results indicate that miR-643 can promote TPC- 1 cell proliferation and inhibit its apoptosis, and its mechanism is closely related to the regulation of the target gene CYP11B1.

\section{Acknowledgments}

Funding: The project was supported by grants from Jiangsu University Clinical Medical Science and Technology Development Fund Project (No. JLY20180011).

\section{Footnote}

Conflicts of Interest: Both authors have completed the ICMJE uniform disclosure form (available at http://dx.doi. org/10.21037/tcr.2020.01.43). The authors have no conflicts of interest to declare.

Ethical Statement: The authors are accountable for all aspects of the work in ensuring that questions related to the accuracy or integrity of any part of the work are appropriately investigated and resolved. The study was approved by the Hospital Medical Ethics Committee (No. EA20180105). Informed consent was obtained from every patient in accordance with the Declaration of Helsinki (as revised in 2013).

Open Access Statement: This is an Open Access article distributed in accordance with the Creative Commons Attribution-NonCommercial-NoDerivs 4.0 International License (CC BY-NC-ND 4.0), which permits the noncommercial replication and distribution of the article with the strict proviso that no changes or edits are made and the original work is properly cited (including links to both the formal publication through the relevant DOI and the license). See: https://creativecommons.org/licenses/by-nc-nd/4.0/.

\section{References}

1. Wiltshire JJ, Drake TM, Uttley L, et al. Systematic Review of Trends in the Incidence Rates of Thyroid Cancer. Thyroid 2016;26:1541-52.

2. Seib CD, Sosa JA. Evolving Understanding of the Epidemiology of Thyroid Cancer. Endocrinol Metab Clin North Am 2019;48:23-35.

3. Bray F, Ferlay J, Soerjomataram I, et al. Global cancer statistics 2018: GLOBOCAN estimates of incidence and mortality worldwide for 36 cancers in 185 countries. CA Cancer J Clin 2018;68:394-424.

4. Chmielik E, Rusinek D, Oczko-Wojciechowska M, et al. Heterogeneity of Thyroid Cancer. Pathobiology 2018;85:117-29.

5. Mansour J, Sagiv D, Alon E, et al. Prognostic value of lymph node ratio in metastatic papillary thyroid carcinoma. J Laryngol Otol 2018;132:8-13.

6. Liu Z, Zeng W, Chen T, et al. A comparison of the clinicopathological features and prognoses of the classical and the tall cell variant of papillary thyroid cancer: a metaanalysis. Oncotarget 2017;8:6222-32.

7. Yakushina VD, Lerner LV, Lavrov AV. Gene Fusions in Thyroid Cancer. Thyroid 2018;28:158-67.

8. Cordioli MI, Moraes L, Cury AN, et al. Are we really at the dawn of understanding sporadic pediatric thyroid carcinoma? Endocr Relat Cancer 2015;22:R311-24.

9. Alamoudi AA, Alnoury A, Gad H. miRNA in tumour metabolism and why could it be the preferred pathway for energy reprograming. Brief Funct Genomics 2018;17:157-69.

10. Gambari R, Brognara E, Spandidos DA, et al. Targeting oncomiRNAs and mimicking tumor suppressor miRNAs: New trends in the development of miRNA therapeutic strategies in oncology (Review). Int J Oncol 2016;49:5-32. 
11. Pishkari S, Paryan M, Hashemi M, et al. The role of microRNAs in different types of thyroid carcinoma: a comprehensive analysis to find new miRNA supplementary therapies. J Endocrinol Invest 2018;41:269-83.

12. López-Rosas I, López-Camarillo C, Salinas-Vera YM, et al. Entamoeba histolytica Up-Regulates MicroRNA-643 to Promote Apoptosis by Targeting XIAP in Human Epithelial Colon Cells. Front Cell Infect Microbiol 2019;8:437.

13. Shen $Y$, Pan $Y, X u ~ L$, et al. Identifying microRNAMrna regulatory network in gemcitabine-resistant cells derived from human pancreatic cancer cells. Tumour Biol 2015;36:4525-34.

14. Schmidt Jensen J, Grønhøj C, Mirian C, et al. Incidence and Survival of Thyroid Cancer in Children, Adolescents, and Young Adults in Denmark: A Nationwide Study from 1980 to 2014. Thyroid 2018;28:1128-33.

15. Bresciani L, Orlandi E, Piazza C. Radiation-induced papillary thyroid cancer: is it a distinct clinical entity? Curr Opin Otolaryngol Head Neck Surg 2019;27:117-22.

16. Patil S, Janardhan D, Suresh S, et al. A Rare Case of Metastasis to the Mandible from the Thyroid Gland: a Case Report and Literature Review. Indian J Surg Oncol 2019;10:422-5.

Cite this article as: Yin H, Shao J. MicroRNA-643 promotes proliferation and inhibits apoptosis of papillary thyroid carcinoma by down-regulating the cytochrome P450 family member 11B1. Transl Cancer Res 2020;9(3):1465-1475. doi: $10.21037 /$ tcr.2020.01.43
17. Izzo L, Pugliese F, Di Poce I, et al. Unusual presentation of metastatic thyroid cancer without a primary: role of diagnostic imaging in a daily clinical setting. G Chir 2019;40:234-7.

18. Rusinek D, Chmielik E, Krajewska J, et al. Current Advances in Thyroid Cancer Management. Are We Ready for the Epidemic Rise of Diagnoses? Int J Mol Sci 2017;18:E1817.

19. Sandiford OA, Moore CA, Du J, et al. Human Aging and Cancer: Role of miRNA in Tumor Microenvironment. Adv Exp Med Biol 2018;1056:137-52.

20. Carroll AP, Goodall GJ, Liu B. Understanding principles of miRNA target recognition and function through integrated biological and bioinformatics approaches. Wiley Interdiscip Rev RNA 2014;5:361-79.

21. Lottrup G, Nielsen JE, Skakkebæk NE, et al. Abundance of DLK1, differential expression of CYP11B1, CYP21A2 and MC2R, and lack of INSL3 distinguish testicular adrenal rest tumours from Leydig cell tumours. Eur J Endocrinol 2015;172:491-9.

22. Cao C, Yang X, Li L, et al. Increased expression of CYP17 and CYP11B1 in subclinical Cushing's syndrome due to adrenal adenomas. Int J Urol 2011;18:691-6. 